\title{
Feasibility of the use of Lentinula edodes mycelium in terbinafine remediation
}

\author{
Agata Kryczyk-Poprawa ${ }^{1}$ (1) $\cdot$ Joanna Piotrowska ${ }^{1} \cdot$ Paweł Żmudzki $^{2} \cdot$ Włodzimierz Opoka $^{1} \cdot$ Bożena Muszyńska $^{3}$
}

Received: 7 February 2020 / Accepted: 21 March 2020 / Published online: 29 March 2020

(c) The Author(s) 2020

\begin{abstract}
A detailed understanding of the fate of xenobiotics introduced into the environment and the mechanisms involved in their biotransformation, biodegradation, and biosorption is essential to improve the efficiency of remediation techniques. Mycoremediation is a form of bioremediation technique that has become increasingly popular in recent years as fungi are known to produce various effective extracellular enzymes that have the potential to neutralize a wide variety of xenobiotics released into the environment. Hence, mycoremediation appears to be a promising technique for the removal of a wide array of toxins and pharmaceutical residues from a damaged environment and wastewater. This study primarily aimed to investigate whether white-rot fungus (Lentinula edodes) can be utilized for the bioremediation of common antifungal agent terbinafine, which is mainly available in the market as powder or cream. The cultures of L.edodes were cultivated in the medium containing terbinafine powder or terbinafine $1 \%$ cream, each at a final concentration of $0.1 \mathrm{mg} \mathrm{mL}^{-1}$. The addition of terbinafine in powder form have a negative effect on biomass growth $(p<0.05)$. The total amount of terbinafine in the dry weight of mycelium after culture was estimated to be $7.63 \pm 0.45 \mathrm{mg}$ and $12.52 \pm 2.46 \mathrm{mg}$ for powder and cream samples, respectively. In addition, there were no traces of terbinafine in any of the samples of medium used for culturing L. edodes after the experimental duration period. The biodegradation products of terbinafine were identified for the first time using UPLC/MS/MS. The biodegradation of terbinafine resulted in the loss of 1-naphthylmethanol, which occurred via oxidative deamination, $N$-demethylation, or tert-butyl group hydroxylation. The results of the study demonstrate that $L$. edodes mycelium can be effectively used for the remediation of terbinafine.
\end{abstract}

Keywords Terbinafine $\cdot$ Biodegradation $\cdot$ Lentinula edodes $\cdot$ Edible mushrooms

\section{Introduction}

Environmental pollution with drugs, pesticides, and personal care products is one of the most challenging issues that the world is facing today. Pharmaceutical compounds play an indispensable role in the treatment and prevention

Agata Kryczyk-Poprawa

agata.kryczyk@uj.edu.pl

1 Department of Inorganic and Analytical Chemistry, Faculty of Pharmacy, Jagiellonian University Medical College, 9 Medyczna Street, 30-688 Kraków, Poland

2 Department of Medicinal Chemistry, Faculty of Pharmacy, Jagiellonian University Medical College, 9 Medyczna Street, 30-688 Kraków, Poland

3 Department of Pharmaceutical Botany, Faculty of Pharmacy, Medical College, Jagiellonian University, Medyczna 9 Street, 30-688 Kraków, Poland of diseases. Active pharmaceutical ingredients (APIs) can enter the environment as a result of various manufacturing processes involved in the production of medicinal products, following medical diagnosis and therapeutic use of pharmaceuticals for the prevention of diseases, or due to disposal of unused medications. According to Executive Agency for Health and Consumers, the annual worldwide consumption of pharmaceutical compounds is estimated to be about 100,000 tonnes (Mudgal et al. 2013). APIs and their metabolites are identified in sewage, surface water, soils, sludge, and air. Their widespread occurrence and potential adverse effects on ecosystems and human health, mainly associated with long-term exposure to low concentrations of pharmaceuticals, are a subject of public concern (Sousa et al. 2012; Patel et al. 2019). After being released into the environment, active metabolites might further undergo degradation to produce toxic end products, which involves complex processes that significantly depend on various factors (Boxall, 2004). 
The investigation concerning the potential risk of drugs present in the environment to the aquatic ecosystems is particularly important (Hilton et al. 2003). Therefore, a better understanding of the fate of medicines in the environment is crucial in attempting to achieve effective drug removal from the surroundings. In response to the growing demand for environmental remediation techniques, numerous studies on water and soil remediation processes, including in situ and ex situ methods, have been carried out. Ex situ methods involve isolation, transportation, and disposal of pollutants at a distant and safe place, but this procedure has been proven to be very expensive. In general, technologies based on physicochemical characteristics of pollutants, such as photocatalytic advanced oxidation processes, membranebased techniques (membrane filtration and reverse osmosis) and chemical and physical adsorption mechanisms, are considered to be highly efficient and cost-effective methods (Kryczyk et al. 2016; Bartolomeu et al. 2018; Warsinger et al. 2018). The recent studies and development in this field are mainly based on the use of nanotechnology, as this novel approach is known to yield better results than the conventional methods. Another possible approach is based on the use of eco-friendly biological processes such as in situ biodegradation. Biological decomposition (bioremediation) is an effective strategy for the removal or transformation of xenobiotics into less toxic forms. Studies conducted worldwide have reported that antifungal drugs (pharmaceutical fungicides) pose a potential threat to the ecosystem. These drugs, particularly those registered for both human and veterinary use, are commonly used as oral or topical agents. This class of drugs could be found in cosmetic products and can be obtained as over-the-counter drugs. In addition, antifungal agents are also widely used in agriculture. Reports of the latest studies establish antifungal drugs as the emerging environmental contaminants (Chen and Ying 2015). The persistence of antifungal drugs in soil and water is highly variable, and it has been observed that some azole antifungals have increased stability and could remain active in some ecological conditions for months (Hof 2001). In Sweden, these agents have been detected in sludge, and the concentrations of clotrimazole, ketoconazole, and econazole are reported to be in the range of $200-1000 \mu \mathrm{g} \mathrm{kg}^{-1}$ dry weight (Lindberg et al. 2010). The occurrence of antifungal agents in the environment has been confirmed in studies conducted locally in many countries (Lindberg et al. 2010; Chen and Ying, 2015; Balakrishna et al. 2017). Terbinafine, an antifungal agent, was detected in the water samples collected from the wells of India at a concentration of more than $1 \mu \mathrm{g} \mathrm{L}^{-1}$ (Fick et al. 2009). Furthermore, this compound was also isolated there from lakes and rivers (Fick et al. 2009). In our previous work, we focused on the mycoremediation of antifungal drugs from the azole group: clotrimazole and bifonazole (Kryczyk-Poprawa et al. 2019). This study primarily aimed to investigate whether white-rot fungus can be effectively utilized for the bioremediation of common antifungal agent terbinafine (in powder and cream forms). Terbinafine hydrochloride, a synthetic allylamine derivative, is widely used for the treatment for fungal infections owing to the fact that it exhibits broad-spectrum antifungal activity and its price is relatively low when compared to other antifungals. Hence, this drug was selected for investigation purpose in the present study. Terbinafine acts by reversible inhibition of squalene epoxidase, an enzyme responsible for ergosterol biosynthesis. This biochemical mechanism is responsible for the fungistatic and fungicidal activity of the drug on fungal mycelia. According to the current medical guidelines, terbinafine is recommended as the first-line treatment for dermatophyte onychomycosis, mainly due to its high efficacy and tolerability properties (Ameen et al. 2014). In 2001, about 1.5 million prescriptions for drugs containing terbinafine were issued in the US alone (Davis et al. 2019). Mushroom cultures could also be utilized to remove xenobiotics from the environment (Kryczyk et al. 2017; Dąbrowska et al. 2018). Lentinula edodes was selected for the current study because of its unique nutritional and healing properties. The Japanese common name of $L$. edodes is "shitake." The fruiting bodies of $L$. edodes contain a number of compounds that contribute to its pharmacological effects, mainly immunomodulatory and anticancer properties. The fruiting bodies of this species are rich in bioactive polysaccharides such as $\beta$-D-glucans (lentinan), monosaccharides, vitamins, and eritadenine (2(R),3( $R$ )-dihydroxy-4-(9-adenyl) butyric acid). These mushrooms also produce many enzymes, such as peroxidase, cellulase, pectinase, xylanase, ligninase, and oxidase. Since $L$. edodes produces oxidative and hydrolytic extracellular enzymes, it can participate in xenobiotic degradation process (Leatham 1985). Additionally, this species was effectively used for the remediation of azole antifungal drugs (Kryczyk-Poprawa et al. 2019). For the above-mentioned reasons, the mycoremediation potential of $L$. edodes mycelia against terbinafine was investigated in in vitro cultures. The medium used for culturing $L$. edodes was enriched with terbinafine powder or terbinafine $1 \%$ cream with the final concentration of the investigated drug being $0.1 \mathrm{mg} \mathrm{mL}^{-1}$. The biodegradation products of terbinafine were analyzed by UPLC/MS/MS method.

\section{Materials and methods}

\section{Reagents}

Terbinafine hydrochloride (pharmaceutical secondary standard) was obtained from Sigma-Aldrich Corp. (St. Louis, MO, USA). HPLC-grade methanol, acetonitrile, formic acid (98\%), chloramphenicol, and dichloromethane were 
obtained from Merck (Darmstadt, Germany). Chemicals like glucose, maltose extract, casein hydrolysate, L-asparagine, adenine, and yeast extract were purchased from Sigma-Aldrich (St. Louis, MO, USA). $\mathrm{NH}_{4} \mathrm{Cl}, \mathrm{KH}_{2} \mathrm{PO}_{4}$, $\mathrm{MgSO}_{4} \cdot 7 \mathrm{H}_{2} \mathrm{O}, \mathrm{CaCl}_{2} \cdot 6 \mathrm{H}_{2} \mathrm{O}, \mathrm{FeCl}_{3}, \mathrm{MnSO}_{4} \cdot \mathrm{H}_{2} \mathrm{O}$, and $\mathrm{ZnSO}_{4} \cdot 7 \mathrm{H}_{2} \mathrm{O}$ were purchased from PPH Golpharm (Kraków, Poland). Water (quadruple-distilled) with a conductivity of less than $1 \mu \mathrm{S} \mathrm{cm}^{-1}$ was obtained using an S2-97A2 distillation apparatus (ChemLand, Stargard Szczeciński, Poland). Terbinafine cream (Terbinafina Ziaja, Ziaja, Poland) was obtained from a local pharmacy store.

\section{Materials for analysis}

For the study, commercially available fruiting bodies of $L$. edodes (Berk.) (Pegler (mushroom) were bought at a supermarket in Poland (2016). The taxonomic identification of the fungus was performed using MycoKey 4.1 software (https ://www.mycokey.com) by an expert named Muszyńska. The representative samples of the material to be used for further studies were stored at the Department of Pharmaceutical Botany, Jagiellonian University Medical College, Kraków, Poland. Fragments of the hymenial part of fruiting bodies were selected to prepare the mycelial culture of $L$. edodes. First, the fragments of the fruiting bodies were mixed with sterile redistilled water and transferred to the BD Sabouraud agar supplemented with chloramphenicol (under laminar airflow). Then, the cultures were incubated in a thermostat (ST500/B/40 Pol-Eko-Apparatus) at $23{ }^{\circ} \mathrm{C} \pm 2{ }^{\circ} \mathrm{C}$ for 2 weeks. Microscopic analysis of the obtained mycelium of $L$. edodes was performed by Muszyńska. It showed the homogeneous nature of hyphae, which was free from contamination with other strains of fungi or bacteria. The mycelia obtained from the solid medium were used to prepare initial cultures that were grown on liquid Oddoux medium (Oddoux 1957). The cultures were shaken on a rotary shaker (ALTEL, Poland) at a rate of $140 \mathrm{rpm}$ and incubated at a temperature of $23{ }^{\circ} \mathrm{C} \pm 2{ }^{\circ} \mathrm{C}$ under 12-h light $(900 \mathrm{~lx}) / 12$-h dark cycle. The cultures of $L$. edodes were maintained for two weeks and later subcultured (Fig. 1).

The obtained $L$. edodes mycelium was analyzed by PCR method. GenBank Accession Number SUB6791903 Seq 1 MN907099 was assigned to the nucleotide sequence determined in this study.

\section{Experimental mycelial cultures of $L$. edodes}

For mycoremediation studies, $25 \mathrm{mg}$ of terbinafine powder or $2.5 \mathrm{~g}$ of terbinafine $1 \%$ cream was added into $250 \mathrm{~mL}$ of sterile liquid medium inoculated with a mycelial culture of L. edodes. Terbinafine $1 \%$ cream (Ziaja) containing isopropyl myristate, cetyl alcohol, stearyl alcohol, sorbitan stearate (Type 50), cetyl palmitate (15), polysorbate 60 ,

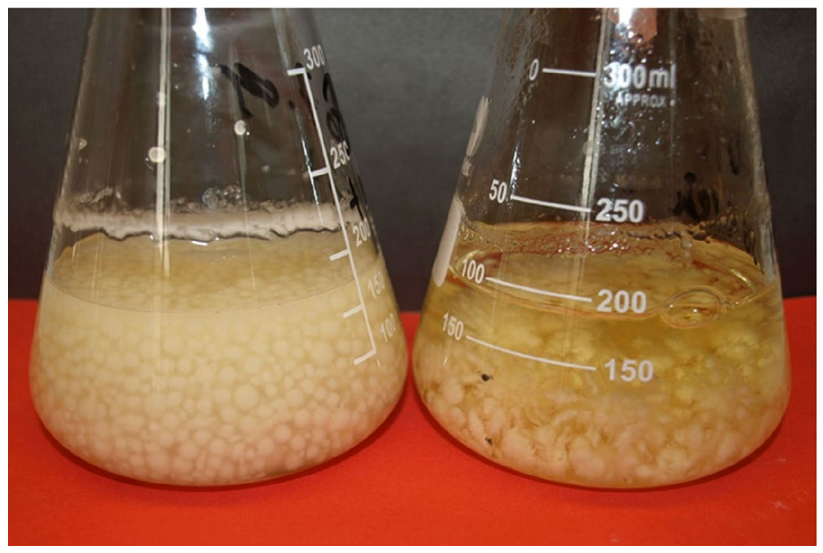

Fig. 1 L. edodes in vitro cultures enriched with the addition of $2.5 \mathrm{~g}$ of terbinafine $1 \%$ cream or $25 \mathrm{mg}$ terbinafine powder per $250 \mathrm{~mL}$ of the Oddoux medium (picture taken by Agata Kryczyk-Poprawa)

benzyl alcohol, sodium hydroxide, and purified water was used. Moreover, control samples were prepared without the addition of terbinafine. After inoculation, the cultures were incubated for 14 days. Then, the biomass of $L$. edodes was separated from the medium, rinsed with redistilled water, and then freeze-dried using a lyophilizer (FreeZone 4.5, Labconco).

\section{RP-HPLC analysis}

Five grams of the lyophilized mushroom culture (mycelium obtained from in vitro cultures) was ground in a mortar and then extracted with a mixture of methanol and dichloromethane $(75: 25, \mathrm{v} / \mathrm{v})$ in an ultrasonic bath at $49 \mathrm{kHz}$ for $30 \mathrm{~min}$ (Sonic-2, Polsonic). The obtained extracts $(300 \mathrm{~mL})$ were evaporated to dryness using a rotary vacuum evaporator at $22{ }^{\circ} \mathrm{C} \pm 2{ }^{\circ} \mathrm{C}$, and then the dried extracts were dissolved in methanol and filtered through a $0.2-\mu \mathrm{m}$ filter. The concentration of terbinafine was determined using RP-HPLC according to the procedure developed by Tagliari (2010). Briefly, the separation was carried out on Hitachi HPLC apparatus (Merck, Tokyo, Japan) equipped with an L-7100 pump, Purospher® RP-C ${ }_{18}(200 \mathrm{~mm} \times 4 \mathrm{~mm}, 5 \mu \mathrm{m})$ column (Merck, Tokyo, Japan), using methanol-water (95:5, v/v) as a mobile phase, and a flow rate of $1 \mathrm{~mL} \mathrm{~min}^{-1}$ with UV detection at $\lambda=254 \mathrm{~nm}$. The qualitative analysis of terbinafine was done by comparing the retention times of the peaks in the samples with the retention times of standard. To confirm the presence of terbinafine in the tested extracts, a standard solution was added to the samples. The presence of the tested compound in the sample was indicated by an increase in the peak height for the appropriate retention time. In addition, MS/MS analysis was also performed. The quantitative analysis of terbinafine was carried out using 
the calibration curve method where the concentration of the standard drug was in the range from 0.01 to $0.1 \mathrm{mg} \mathrm{mL}^{-1}$.

\section{UPLC/MS/MS analysis}

About $200 \mu \mathrm{L}$ of the sample was evaporated and diluted in $300 \mu \mathrm{L}$ of water with the addition of $1 \%$ formic acid. The samples were applied to the SPE cartridges (Waters Oasis HLB Cartridge, USA) and washed with $1 \mathrm{~mL}$ of water, followed by the addition of $1 \mathrm{~mL}$ of methanol-water $(50: 50, \mathrm{v} / \mathrm{v})$ mixture, and then eluted with $1 \mathrm{~mL}$ of methanol. The prepared samples were injected into the UPLC/MS/ MS system for analysis. The UPLC/MS/MS system consisted of a Waters ACQUITY® UPLC® (Waters Corporation, Milford, MA, USA) coupled to a Waters TQD mass spectrometer (electrospray ionization mode ESI-tandem quadrupole). Chromatographic separation was carried out using Acquity UPLC BEH (bridged ethyl hybrid) $\mathrm{C} 18$ column, $2.1 \times 100 \mathrm{~mm}$ and $1.7 \mu \mathrm{m}$ particle size, equipped with Acquity UPLC BEH C18 VanGuard pre-column, $2.1 \times 5 \mathrm{~mm}$ and $1.7 \mu \mathrm{m}$ particle size. The column was maintained at $40{ }^{\circ} \mathrm{C}$, and eluted under gradient conditions using $95-0 \%$ of eluent $\mathrm{A}$ for $10 \mathrm{~min}$, at a flow rate of $0.3 \mathrm{~mL} \mathrm{~min}{ }^{-1}$. Eluent $\mathrm{A}$ was composed of water/formic acid $(0.1 \%, v / v)$, and eluent B was composed of acetonitrile/formic acid $(0.1 \%, \mathrm{v} / \mathrm{v})$. Chromatograms were recorded using Waters e $\lambda$ PDA detector. Spectra were evaluated in $200-700 \mathrm{~nm}$ range with $1.2-\mathrm{nm}$ resolution at a sampling rate of 20 points/s. Mass spectrometry detection settings of Waters TQD mass spectrometer were as follows: source temperature $150{ }^{\circ} \mathrm{C}$, desolvation temperature $350{ }^{\circ} \mathrm{C}$, desolvation gas flow rate $600 \mathrm{~L} \mathrm{~h}^{-1}$, cone gas flow rate $100 \mathrm{~L} \mathrm{~h}^{-1}$, capillary potential $3.00 \mathrm{kV}$, and cone potential $6 \mathrm{~V}$. Nitrogen gas was used as both nebulizing and drying agent. The data were recorded in the scan mode ranging from 50 to $1000 \mathrm{~m} / z$ in time intervals of $0.5 \mathrm{~s}$; eight scans were summed up to obtain the final spectrum. The data acquisition software used was MassLynx V 4.1 (Waters).

\section{Statistical analysis}

Statistical analyses were conducted using Microsoft Excel 2010 and GraphPad Prism v3.02 (GraphPad Software, San Diego, CA, USA). Three replicates were used for each sample. Results are expressed as means and standard deviation (SD). One-way analysis of variance test with Tukey's post hoc test of multiple comparisons or Mann-Whitney's nonparametric test was applied. Values were considered significantly different at $p<0.05$. In silico toxicity risk profile of biotransformation products was predicted using OSIRIS Property Explorer (access: https://www.organic-chemistry. org/prog/peo/).

\section{Results and discussion}

Biodegradation is a biochemical process that leads to the degradation and recycling of xenobiotics. This process primarily involves bacteria, fungi, and algae, and their activity leads to the decomposition of the initial substance into simpler compounds, which do not usually exhibit toxic effects. Due to increasing risks associated with environmental pollution, novel methods showing increased bioremediation efficiency and new possibilities of its application are being explored. It should be emphasized that mycoremediation has become an increasingly popular method for the removal of pollutants from the environment, and, therefore, studies to identify new fungal species with the potential to degrade xenobiotic compounds to evaluate the effectiveness of mushroom-based remediation techniques are highly warranted. The knowledge regarding the biodegradation and biotransformation of antifungal drugs by the mycelia obtained from in vitro cultures is one of the essential elements that prompted us to select white-rot fungus, L. edodes, for mycoremediation of the xenobiotic in our study.

Terbinafine is metabolized in animals and humans (Schatz et al. 1986). It is absorbed from the gastrointestinal tract in more than $70 \%$ of the individuals after oral administration, following intake of $250 \mathrm{mg}$ of the drug, and its maximum plasma concentration is noticed within $1.5 \mathrm{~h}$ (Elewski et al. 2005). The drug accumulates in adipose tissue and keratin-rich tissues (dermis, hair, and nails), wherein effective fungicidal concentration is observed. It is metabolized in the presence of CYP isoenzymes to metabolites that lack antifungal activity. Fifteen liver metabolites of terbinafine have been described during preclinical and clinical studies (Iverson and Uetrecht, 2001). The hepatic metabolism of terbinafine mainly takes place through $N$-demethylation, deamination, and oxidation of the alkyl side chain (Vickers et al. 1999). The present study was undertaken to compare the biodegradation products of terbinafine, its hepatic metabolites, and its photoproducts formed under UVA irradiation. The identification of the degradation products of terbinafine in the mycelium obtained from in vitro cultures of $L$. edodes was performed on the basis of UPLC/MS/MS analysis. The proposed structures of the degradation products of terbinafine are shown in Fig. 2. Two biodegradation products of terbinafine (TP-1 and TP-7) have been previously described. TP-1 and TP-7 were formed during degradation of terbinafine in the presence of UVA irradiation (Kryczyk-Poprawa et al. 2019). The biodegradation of terbinafine resulted in the loss of 1-naphthylmethanol, which occurred via oxidative deamination, $N$-demethylation, or tert-butyl group hydroxylation. The structures of potential 

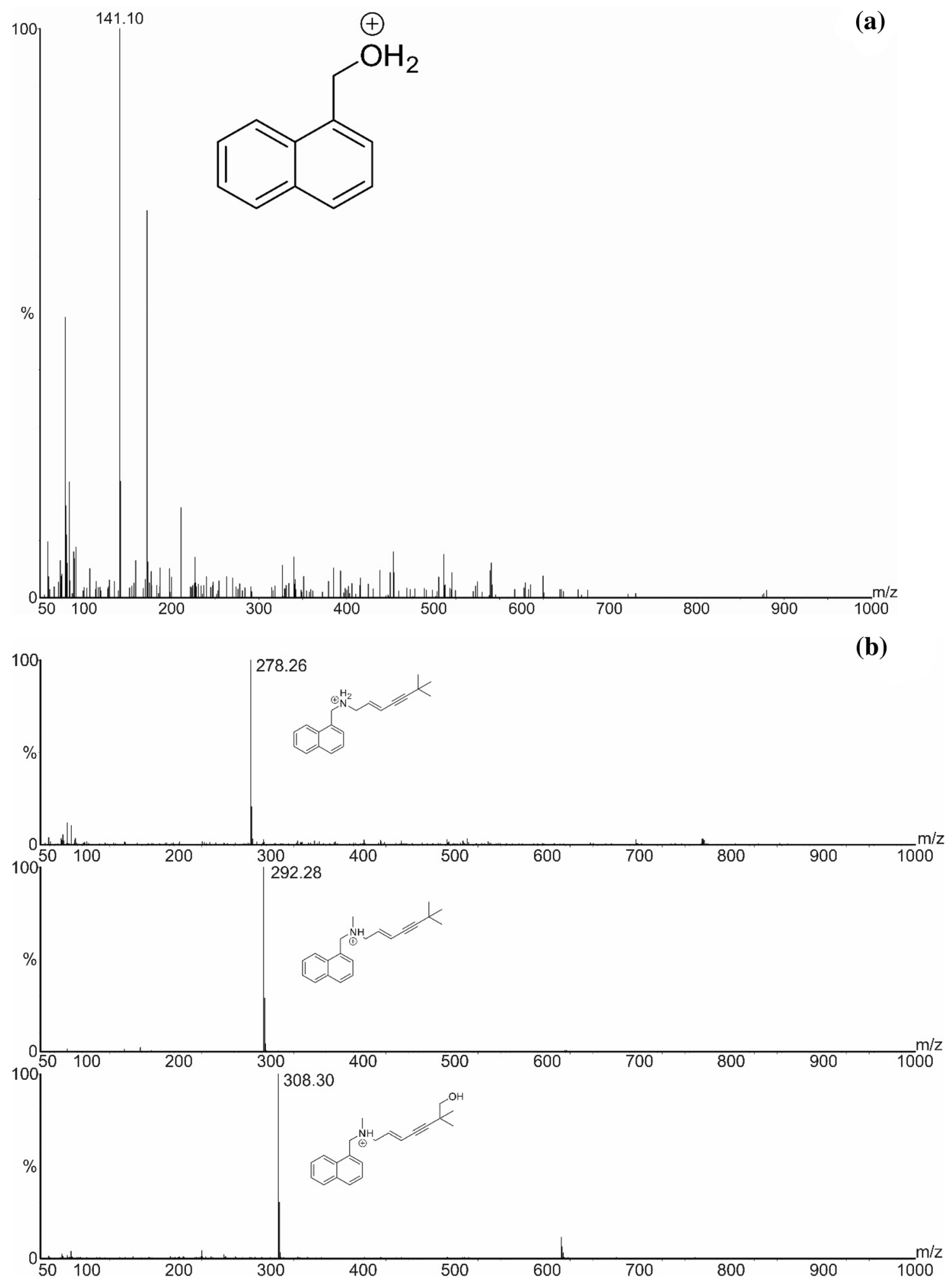

Fig. 2 MS/MS spectra of biodegradation products of terbinafine: a TP-1 and b TP-12, terbinafine, and TP-7

products of terbinafine degradation identified in the mycelium obtained from in vitro cultures of $L$. edodes showed the following properties: TP-1 produced a $[\mathrm{M}+\mathrm{H}]^{+}$at $\mathrm{m} / \mathrm{z}$
141.1, TP-7 produced a $[\mathrm{M}+\mathrm{H}]^{+}$at $m / z$ 308, and TP-12identified for the first time-produced a $[\mathrm{M}+\mathrm{H}]^{+}$at $m / z$ 278. TP-12 was formed as a result of N-demethylation 
of terbinafine to desmethyl terbinafine. This compound further forms 6, 6-dimethyl-2-hepten-4-ynal in the livera compound that can initiate idiosyncratic drug-induced liver toxicity (Iverson and Uetrecht 2001; Barnette et al. 2019). After previous application of $\beta$-glucuronidase hydrolysis in urine and plasma, the metabolite TP-7 was determined which was also identified in mycelium (Zehender et al. 1995).

Many ecotoxicological test systems have been introduced to study the effect of xenobiotics on the aquatic organisms (Küster and Adler 2014). The toxicity studies of terbinafine were carried out in the green algae Pseudokirchneriella subcapitata. Terbinafine is listed in the Swedish Environmental Classification of Pharmaceuticals as the only antifungal drug that poses moderate risk to the environment alongside other compounds such as chlorhexidine, mycophenolic acid and norethisterone (Graae and Pålsson 2018). The harmful effects of terbinafine hydrochloride have been described in Pharmacopeia, in Safety Data Sheet Sect. 2 (Hazards Identification), where it is mentioned that terbinafine is very toxic for aquatic organisms (British Pharmacopoeia Commission 2015). There are also data on the genotoxic effects of terbinafine (Chien et al. 2012; Tolomeotti et al. 2015). We next aimed to evaluate the toxicity-risk predictions of terbinafine and its degradation products using OSIRIS Property Explorer. The toxicity risk assessment was done based on their ability to induce mutagenicity, tumorigenicity, irritation, and reproductive effects (Table 1). The in silico experiments revealed that terbinafine and its degradation products theoretically displayed high tumorigenic activity, which can be attributed to the presence of 1-naphthyl fragment. TP-1, a product of oxidative deamination, led to the formation of 1-naphthylmethanol which demonstrated medium mutagenic activity; hence, further studies aiming to evaluate its safety profile are required.

Figure 3 shows a comparison of dry biomass obtained from in vitro cultures of $L$. edodes enriched with terbinafine (2.5 $\mathrm{g}$ of terbinafine $1 \%$ cream or $25 \mathrm{mg}$ of terbinafine powder) and control (without the addition of terbinafine). The dry weight of mycelium showed statistically significant differences between the control and the fungal sample supplemented with terbinafine powder $(p<0.05)$. These differences may result from terbinafine concentration in the

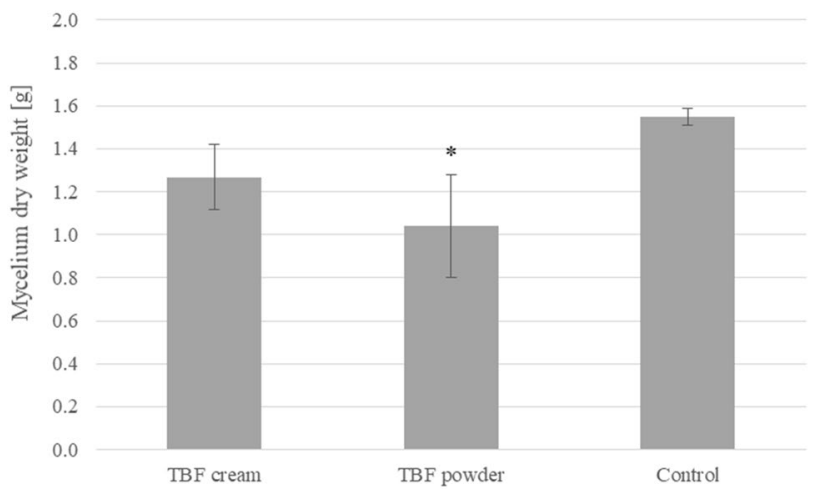

Fig. 3 Comparison of dry matter of Lentinula edodes mycelium obtained from in vitro cultures enriched with the addition of $2.5 \mathrm{~g}$ of terbinafine $1 \%$ cream or $25 \mathrm{mg}$ of terbinafine powder per $250 \mathrm{~mL}$ of the medium. Statistical analysis: one-way analysis of variance (ANOVA), followed by Tukey's test. Significant difference compared to the control group (without terbinafine addition): $* p<0.05, n=3$

medium. The addition of terbinafine in the form of powder, which shows poor solubility in the water, probably allows for immediate availability of the drug for the remediation process, which could have a negative effect on biomass growth. In the case of cream, the form of the drug itself slows down the rate of release of terbinafine into the medium and, thus, limits its availability for the remediation process.

The next stage of the study was to compare of the amount of the tested compound present per $1 \mathrm{~g}$ of dry matter of the mycelium in different samples after the experimental duration period. The calculation took into account the mass of dry mycelium obtained after 4 weeks of cultivation. Figure 4 presents terbinafine concentrations in mycelium obtained from in vitro cultures of L. edodes on Oddoux medium enriched with $25 \mathrm{mg}$ of terbinafine or $2.5 \mathrm{~g}$ of terbinafine $1 \%$ cream per $250 \mathrm{~mL}$ of the medium, respectively. There were no statistically significant $(p<0.05)$ differences in the concentration of terbinafine in the mycelium cultured in the medium containing different forms of the drug. The content of terbinafine in the medium was below the limit of quantification in all the analyzed samples, which indicates that the drug was successfully degraded by the fungus. Therefore, mycoremediation can serve as an alternative approach to traditional remediation techniques.
Table 1 Toxicity risks of terbinafine and its biodegradation products determined in L. edodes mycelium obtained from in vitro cultures in which media were enriched with terbinafine and predicted by OSIRIS Property Explorer

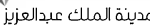

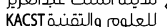

\begin{tabular}{|c|c|c|c|c|}
\hline Compound & Mutagenic & Tumorigenic & Irritant & $\begin{array}{l}\text { Repro- } \\
\text { ductive } \\
\text { effects }\end{array}$ \\
\hline Terbinafine & - & High-risk fragment (1-naphthyl) & - & - \\
\hline TP-1 & $\begin{array}{l}\text { Medium-risk fragment } \\
\text { (1-naphthylmethanol) }\end{array}$ & High-risk fragment (1-naphthyl) & - & - \\
\hline ТP-7 & - & High-risk fragment (1-naphthyl) & - & - \\
\hline TP-12 & - & High-risk fragment (1-naphthyl) & - & - \\
\hline
\end{tabular}




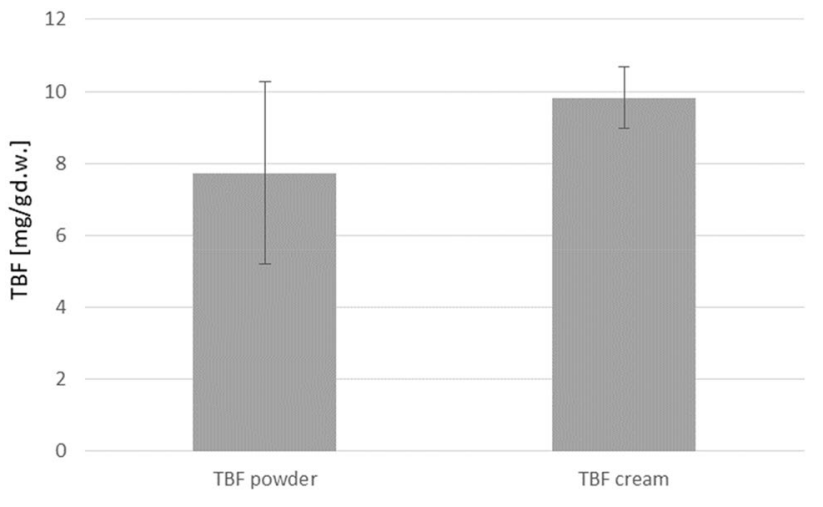

Fig. 4 Terbinafine content in $1 \mathrm{~g}$ of dry matter obtained from in vitro cultures of Lentinula edodes enriched with the addition of $25 \mathrm{mg}$ of terbinafine powder or $2.5 \mathrm{~g}$ of terbinafine $1 \%$ cream per $250 \mathrm{~mL}$ of the medium. Statistical analysis: Mann-Whitney' nonparametric test: $\mathrm{NS}, n=3$

The total content of the investigated drug in L. edodes mycelium was calculated on the basis of the terbinafine content determined in $1 \mathrm{~g}$ of dry matter obtained from $L$. edodes in vitro cultures and the dry weight of the examined mycelium. The total terbinafine content in L. edodes mycelium, obtained from in vitro cultures, in which media were enriched with terbinafine in powder or cream form at a concentration of $0.1 \mathrm{mg} \mathrm{mL}^{-1}$ was $7.63 \pm 0.45 \mathrm{mg}$ and $12.52 \pm 2.46 \mathrm{mg}$, respectively. The use of two different forms of the drug allowed to demonstrate the impact of drug form on the process of remediation by L. edodes. A higher amount of terbinafine was determined in the dry matter obtained from in vitro cultures of $L$. edodes supplemented with $1 \%$ cream. In the case of powder, the amount of terbinafine determined in the mycelium obtained from in vitro cultures of $L$. edodes and its absence in the medium indicate a more advanced process of biodegradation.

\section{Conclusion}

Many reports have been published concerning the role of antifungal agents in causing environmental pollution. The present study focuses on the evaluation of the potential of $L$. edodes to accumulate or/and degrade the common antifungal drug terbinafine. In vitro culturing of L. edodes in the presence of the drug, thanks to the application of reproducible culture conditions, and examining the amount of the drug in the mycelium after culture allowed for the assessment of the remediation process of the examined compound. The study results demonstrates that $L$. edodes can be effectively used for the bioremediation of terbinafine.

Funding This work was supported by the statutory fund N42/ DBS/000030 from Jagiellonian University Medical College.

\section{Compliance with ethical standards}

Conflict of interest On behalf of all authors, the corresponding author states that there is no conflict of interest.

Open Access This article is licensed under a Creative Commons Attribution 4.0 International License, which permits use, sharing, adaptation, distribution and reproduction in any medium or format, as long as you give appropriate credit to the original author(s) and the source, provide a link to the Creative Commons licence, and indicate if changes were made. The images or other third party material in this article are included in the article's Creative Commons licence, unless indicated otherwise in a credit line to the material. If material is not included in the article's Creative Commons licence and your intended use is not permitted by statutory regulation or exceeds the permitted use, you will need to obtain permission directly from the copyright holder. To view a copy of this licence, visit http://creativecommons.org/licenses/by/4.0/.

\section{References}

Ameen M, Lear JT, Madan V, Mohd Mustapa MF, Richardson M (2014) British association of dermatologists' guidelines for the management of onychomycosis 2014. Br J Dermatol. https://doi. org/10.1111/bjd.13358

Balakrishna K, Rath A, Praveenkumarreddy Y, Guruge KS, Subedi B (2017) A review of the occurrence of pharmaceuticals and personal care products in Indian water bodies. Ecotoxicol Environ Saf. https://doi.org/10.1016/j.ecoenv.2016.11.014

Barnette DA, Davis MA, Flynn N, Pidugu AS, Swamidass SJ, Miller GP (2019) Comprehensive kinetic and modeling analyses revealed CYP2C9 and 3A4 determine terbinafine metabolic clearance and bioactivation. Biochem Pharmacol. https://doi.org/10.1016/j. bcp.2019.113661

Bartolomeu M, Neves MGPMS, Faustino MAF, Almeida A (2018) Wastewater chemical contaminants: remediation by advanced oxidation processes. Photochem Photobiol Sci. https://doi. org/10.1039/c8pp00249e

Boxall ABA (2004) The environmental side effects of medication. EMBO Rep. https://doi.org/10.1038/sj.embor.7400307

British Pharmacopoeia Commission (2015) Retrieved from British Pharmacopoeia website: https://www.pharmacopoeia.com/Catal ogue/Preview?uri=\%2Fcontent $\% 2$ Ffile $\% 2$ Fproducts $\% 2$ Fhealthan dsafety\%2FCat_1159_GB.pdf. Accessed 15 Dec 2019

Chen ZF, Ying GG (2015) Occurrence, fate and ecological risk of five typical azole fungicides as therapeutic and personal care products in the environment: a review. Environ Int. https://doi. org/10.1016/j.envint.2015.07.022

Chien MH, Lee TS, Kao C, Yang SF, Lee WS (2012) Terbinafine inhibits oral squamous cell carcinoma growth through anti-cancer cell proliferation and anti-angiogenesis. Mol Carcinog. https://doi. org/10.1002/mc. 20800

Dąbrowska M, Muszyńska B, Starek M, Żmudzki P, Opoka W (2018) Degradation pathway of cephalosporin antibiotics by in vitro cultures of Lentinula edodes and Imleria badia. Int Biodeterior Biodegrad. https://doi.org/10.1016/j.ibiod.2017.11.014

Davis MA, Barnette DA, Flynn NR, Pidugu AS, Swamidass SJ, Boysen G, Miller GP (2019) CYP2C19 and 3A4 dominate metabolic clearance and bioactivation of terbinafine based on computational and experimental approaches. Chem Res Toxicol. https://doi. org/10.1021/acs.chemrestox.9b00006 
Elewski B, Tavakkol A (2005) Safety and tolerability of oral antifungal agents in the treatment of fungal nail disease: a proven reality. Ther Clin Risk Manag 1(4):299-306

Fick J, Söderström H, Lindberg RH, Phan C, Tysklind M, Larsson DGJ (2009) Contamination of surface, ground, and drinking water from pharmaceutical production. Environ Toxicol Chem. https://doi. org/10.1897/09-073.1

Graae AL, Pålsson LÖ (2018) Self-declarations of environmental classification at Fass.se. Retrieved from https://www.ivl.se/downl oad/18.2299af4c16c6c7485d0247d/1570535585458/B2361.pdf. Accessed 15 Dec 2019

Hilton M, Thomas K, Ashton D (2003) Targeted Monitoring Programme for Pharmaceuticals in the Aquatic Environment. Retrieved from https://www.freshwaterlife.org/projects/media /projects/images/5/83919_ca_object_representations_media 596_original.pdf. Accessed 15 Dec 2019

Hof H (2001) Critical annotations to the use of azole antifungals for plant protection. Antimicrob Agents Chemother. https://doi. org/10.1128/AAC.45.11.2987-2990.2001

Iverson SL, Uetrecht JP (2001) Identification of a reactive metabolite of terbinafine: insights into terbinafine-induced hepatotoxicity. Chem Res Toxicol. https://doi.org/10.1021/tx0002029

Kryczyk A, Żmudzki P, Hubicka U (2016) Determination of itraconazole and its photodegradation products with kinetic evaluation by ultra-performance liquid chromatography/tandem mass spectrometry. Biomed Chromatogr. https://doi.org/10.1002/bmc.3747

Kryczyk A, Piotrowska J, Sito M, Sulkowska-Ziaja K, Dobosz K, Opoka W, Muszynska B (2017) Remediation capacity of cd and $\mathrm{pb}$ ions by mycelia of Imleria badia, Laetiporus sulphureus, and Agaricus bisporus in vitro cultures. J Environ Sci Health B. https ://doi.org/10.1080/03601234.2017.1330068

Kryczyk-Poprawa A, Żmudzki P, Koczurkiewicz P, Pękala E, Hubicka U (2019) Photostability of terbinafine under UVA irradiation: the effect of UV absorbers. Photochem Photobiol. https://doi. org/10.1111/php.13075

Küster A, Adler N (2014) Pharmaceuticals in the environment: scientific evidence of risks and its regulation. Philos Trans R Soc Lond B Biol Sci. https://doi.org/10.1098/rstb.2013.0587

Leatham GF (1985) Extracellular enzymes produced by the cultivated mushroom Lentinus edodes during degradation of a lignocellulosic medium. Appl Environ Microbiol 50:859-867

Lindberg RH, Fick J, Tysklind M (2010) Screening of antimycotics in Swedish sewage treatment plants-waters and sludge. Water Res. https://doi.org/10.1016/j.watres.2009.10.034
Mudgal S, De Toni A, Lockwood S, Salès K, Backhaus T, Sorensen BH (2013) Study on the environmental risks of medicinal products. Retrieved from https://ec.europa.eu/health/sites/health/files/files/ environment/study_environment.pdf. Accessed 15 Dec 2019

Oddoux L (1957) Recherches sur les Mycéliumssecondaires des Homobasidiésen Culture Pure. Imprimerie de Trevoux, Lyon

Patel M, Kumar R, Kishor K, Mlsna T, Pittman CU, Mohan D (2019) Pharmaceuticals of emerging concern in aquatic systems: Chemistry, occurrence, effects, and removal methods. Chem Rev. https ://doi.org/10.1021/acs.chemrev.8b00299

Schatz F, Haberl H, Battig F, Jobstmann D, Schulz G, Nefzger M, Czok R, Nikiforov A (1986) Major routes of naftifine biotransformation in laboratory animals and man. Arzneimittelforschung $36: 248-255$

Sousa MA, Gonçalves C, Vilar VJP, Boaventura RAR, Alpendurada MF (2012) Suspended $\mathrm{TiO}_{2}$-assisted photocatalytic degradation of emerging contaminants in a municipal WWTP effluent using a solar pilot plant with CPCs. Chem Eng J. https://doi.org/10.1016/j. cej.2012.05.060

Tagliari MP, Kuminek G, Borgmann SHM, Bertol CD, Cardoso SG, Stulzer HK (2010) Terbinafine: optimization of a LC method for quantitative analysis in pharmaceutical formulations and its application for a tablet dissolution test. Quim Nova. https://doi. org/10.1590/S0100-40422010000800029

Tolomeotti D, De Castro-Prado MAA, De Santanna JR, Martins ABT, Della-Rosa VA (2015) Genotoxic evaluation of terbinafine in human lymphocytes in vitro. Drug Chem Toxicol. https://doi. org/10.3109/01480545.2014.959174

Vickers AEM, Sinclair JR, Zollinger M, Heitz F, Glänzel U, Johanson L, Fischer V (1999) Multiple cytochrome P-450s involved in the metabolism of terbinafine suggest a limited potential for drugdrug interactions. Drug Metab Dispos 27:1029-1038

Warsinger DM, Chakraborty S, Tow EW, Plumlee MH, Bellona C, Loutatidou S, Karimi L, Mikelonis AM, Achilli A, Ghassemi A, Padhye LP, Snyder SA, Curcio S, Vecitis C, Arafat HA, Lienhard JH (2018) A review of polymeric membranes and processes for potable water reuse. Prog Polym Sci. https://doi.org/10.1016/j. progpolymsci.2018.01.004

Zehender H, Denouël J, Roy M, Le Saux L, Schaub P (1995) Simultaneous determination of terbinafine (Lamisil) and five metabolites in human plasma and urine by high-performance liquid chromatography using on-line solid-phase extraction. J Chromatogr B Biomed Appl. https://doi.org/10.1016/0378-4347(94)00483-L 